\title{
Respiratory abnormalities due to craniovertebral junction compression in rheumatoid disease
}

\author{
R S Howard, F Henderson, N P Hirsch, J M Stevens, B E Kendall, H A Crockard
}

\begin{abstract}
Objectives-To assess the extent and severity of respiratory insufficiency associated with severe rheumatoid atlantoaxial dislocation and its relation to compression of the neuraxis.

Methods-Twelve patients with severe atlantoaxial dislocation due to rheumatoid disease were studied. Detailed clinical, CT myelography and respiratory assessment including nocturnal oximetry, were performed on all patients.

Results-All patients were severely disabled by their underlying disease but none had symptoms of hypoventilation. All the patients with $\mathrm{C} 1$ compression had myelopathic features. Those with medullary deformation (moulding and/or stretch) had abnormal noctural oximetry whilst no significant desaturations were seen in the remaining patients. Postoperative studies showed resolution of noctural desaturations.

Conclusion-This study suggests that clinically unsuspected respiratory insufficiency may be common in patients with severe medullary compression associated with rheumatoid atlantoaxial dislocation. It emphasises the importance of careful respiratory monitoring including nocturnal oxygen saturation in patients with major atlantoaxial dislocation due to rheumatoid disease.
\end{abstract}

(Ann Rheum Dis 1994; 53: 134-136)

The Harris Unit, The National Hospital for Neurology and Neurosurgery, Queen Square, London, United Kingdom R S Howard F Henderson N P Hirsch

J M Stevens

BE Kendall

H A Crockard

Correspondence to:

Dr R S Howard,

Dr R S Howard,

The National Hospital

for Neurology and

Square, London

Square, Londo

United Kingdom.

United Kingdom.

3 November 1993 cervical myelopathy, ${ }^{4}$ upward translocation of the odontoid process, ${ }^{5}$ severe cord compression $^{6}$ and intermittent neurological disturbances are all poor prognostic features. The poor prognosis of conservatively treated patients with craniocervical compression had led to the development of various operative strategies. ${ }^{7}$ It is well recognised that apnoea and sudden death may occur as a consequence of medullary compression disorders in the region of the foramen magnum. ${ }^{8}$ The extent to which hypoventilation and apnoea contribute to the high mortality of rheumatoid atlantoaxial dislocation with neural involvement remains unknown.

\section{Methods}

Twelve consecutive patients with atlantoaxial dislocation due to rheumatoid disease, not necessarily with respiratory symptoms, were studied as part of an ongoing study of 255 patients undergoing odontoidectomy and posterior fixation. Detailed clinical, CT myelography and respiratory assessment including nocturnal oximetry, were performed in all patients. Six patients were studied postoperatively. Oxygenation was measured continuously using an Ohmeda $3700 \mathrm{e}$ pulse oximeter and transferred for subsequent computer analysis. The overnight baseline oxygen saturation $\left(\mathrm{SpO}_{2}\right)$ and the number of dips in $\mathrm{SpO}_{2}>4 \%$ lasting longer than ten seconds were calculated and expressed per hour of recording. ${ }^{9}$ The presence of sleep was documented by the nurse observing the patient but polysomnography was not performed because of the severity of the underlying disability.

Eleven patients were investigated by plain radiography and water soluble CT myelography and one with MRI. The CT technique was as follows: slice thickness of $1.5 \mathrm{~mm}$ performed sequentially across the entire craniocervical junction from external auditory meatus to $\mathrm{C} 3$ with both flexion and extension of the head, and $5 \mathrm{~mm}$ thick slices were made with $2 \mathrm{~mm}$ overlap to examine the entire cervical spine from C2 to T1. Multiple sagittal, coronal and axial reformatted images were prepared from this data. The following measurements were made: minimum midsagittal diameter of the cord and medulla oblongata when either or both appeared compressed (cord cross sectional area, CSA), the atlanto-dental distance (ADI), and the degree of ventral migration of the dens (VMD) using the method described by RedlundJohnell. ${ }^{10}$ The clivoaxial angle (CAA) was measured, defined as the obtuse angle made between a line drawn from the posterior 

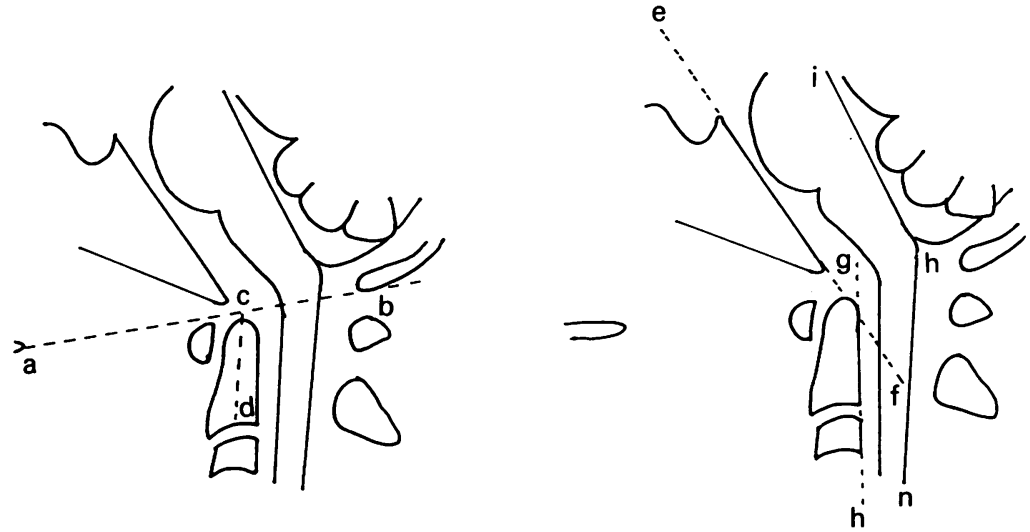

Figure 1 Diagrams to show some of the measurements referred to in the text. Palatooccipital line ( $a-b)$; vertical migration of the dens (VMD) (c-d); the clivo-axial angle $(C A A)$ was the obtuse angle between lines $e-f$ and $g-h$; the cervical flexure of the neural axis was the obtuse angle between lines $i-h$ and $h-n$.

surface of the clivus intersecting with the upward projection of a line drawn along the posterior surface of the body of the axis.

Finally the degree of kyphosis of the lower brainstem or cervical cord was estimated by assessing the cervical flexure of the neural axis. Precise measurement of this angle was not possible in most studies because not enough of the upper rhombencephalon was visible. An angle of greater than $135^{\circ}$ was defined as normal. These distances and angles are illustrated in figure 1. Moulding of the medulla oblongata by the vertically migrated dens was assessed visually and scored as present or absent.

\section{Results}

There were 11 women and one man, the mean age of presentation was $53 \cdot 7(50-78)$ years and the mean duration of RA was 22.9 (7-50) years. Eleven patients presented with neck pain, three with limb weakness and one with dysphagia. One patient was breathless at presentation but no respiratory symptoms were present in the remaining patients. There were signs of cord compression in four patients and bulbar weakness was present in a further patient with coexisting downward hindbrain herniation. There was a past history of mild obstructive airways disease in two patients and bronchiectasis in one. Ten patients were taking disease modifying drugs (Gold and penicillamine) and seven corticosteroids, all took non steroidal anti-inflammatory drugs.

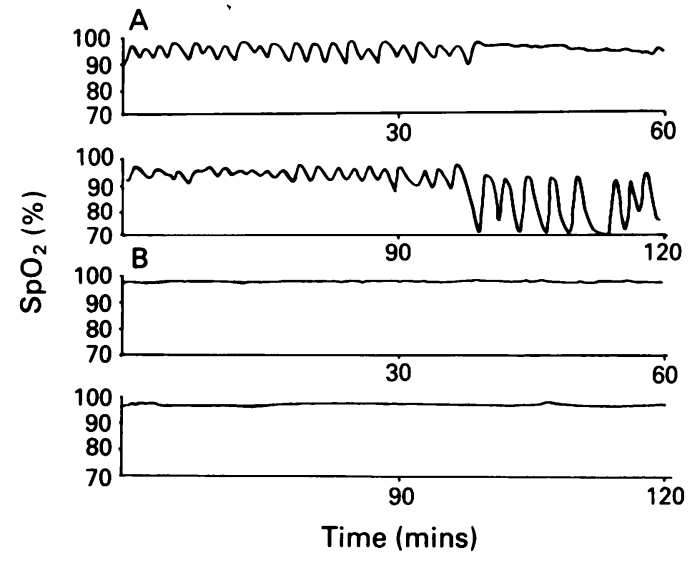

Figure 2 Nocturnal oximetry tracing $(A)$ pre- and $(B)$ postoperatively for the first two hours of sleep. Trace $(A)$ shows frequent desaturations of variable duration and severity. These have resolved completely postoperatively.

Nine took analgesics containing codeine or dextropropoxyphene and six were taking hypnotics; none took opiates. Patients remained on the same medication during preand postoperative studies. None of the patients smoked. CT myelographic findings are described in the table. Three patients had evidence of underlying pulmonary disease, such as, bronchiectasis, or chronic obstructive airways disease but in most of the other patients spirometric indices were significantly below predicted values. Noctural oximetry showed $>5$ hypoxic dips/hour in four patients. In two other patients there were intermittent prolonged periods of sustained desaturations lasting up to 20 minutes, suggestive of alveolar hypoventilation. The patient with bronchiectasis also had frequent and severe dips below a low baseline. Five patients (including the patient with chronic obstructive airways disease) had normal overnight oximetry. In general, the minimum value of $\mathrm{SpO}_{2}$ was lowest in those patients with the most frequent dips and the longest duration $4 \%$ below baseline.

There was clinical evidence of myelopathy in the four patients with the most severe cervical cord compression and only one of these had moulding or abnormal kyphos of the medulla oblongata. None of the patients with predominant medullary involvement had myelopathy, regardless of severity, and only the patient with coexisting hindbrain descent had cranial nerve involvement. Three patients with myelopathy had mild abnormalities of noctural

Radiological features

\begin{tabular}{|c|c|c|c|c|c|c|c|c|}
\hline Patient & $\begin{array}{l}\text { Site of maximal } \\
\text { compression }\end{array}$ & $\begin{array}{l}\text { Other sites of } \\
\text { compression }\end{array}$ & $\begin{array}{l}\text { Abnormal } \\
\text { cervical } \\
\text { flexure }\end{array}$ & Pannus & $\begin{array}{l}\text { Molding of } \\
\text { medulla }\end{array}$ & $\begin{array}{l}\text { cord } \\
C S A\left(\mathrm{~mm}^{2}\right)\end{array}$ & $V M D\left(m^{2}\right)$ & $\begin{array}{l}C C A \\
()\end{array}$ \\
\hline $\begin{array}{r}1 \\
2 \\
3 \\
4 \\
5 \\
6 \\
7 \\
8 \\
9 \\
10 \\
11 \\
12\end{array}$ & $\begin{array}{l}\text { Medulla } \\
\text { C1 (ant and post) } \\
\text { None } \\
\text { Medulla } \\
\text { C1 (on flexion) } \\
\text { Medulla } \\
\text { C1 (on flexion) } \\
\text { C1 (ant and post) } \\
\text { C1 (ant and post) } \\
\text { C1 (minimal) } \\
\text { None } \\
\text { C1 (ant and post) }\end{array}$ & $\begin{array}{l}\text { C1 (ant and post) } \\
\text { Medulla } \\
\text { Medulla } \\
\text { C1 (coexisting ACM) } \\
\text { Medulla } \\
\text { Medulla }\end{array}$ & $\begin{array}{l}+ \\
+ \\
0 \\
+ \\
+ \\
+ \\
0 \\
+ \\
0 \\
0 \\
0 \\
0\end{array}$ & $\begin{array}{l}+ \\
0 \\
0 \\
0 \\
0 \\
0 \\
+ \\
0 \\
0 \\
0 \\
0 \\
+\end{array}$ & $\begin{array}{l}+ \\
+ \\
0 \\
0 \\
0 \\
+ \\
+ \\
+ \\
0 \\
0 \\
0 \\
+\end{array}$ & $\begin{array}{l}60 \\
36 \\
70 \\
68 \\
30 \\
\\
37 \\
37 \\
75 \\
68 \\
\\
70\end{array}$ & $\begin{array}{r}21 \\
20 \\
7 \\
24 \\
16 \\
16 \\
12 \\
21 \\
9 \\
12 \\
15\end{array}$ & $\begin{array}{r}90 \\
154 \\
140 \\
122 \\
120 \\
135 \\
150 \\
125 \\
150 \\
135 \\
150\end{array}$ \\
\hline
\end{tabular}


oximetry although none had more than five hypoxic dips/hour.

All patients with medullary deformation (moulding and/or abnormal kyphos) had abnormal noctural oximetry. No significant desaturations were seen in the remaining patients. Similarly the presence of marked VMD $<15 \mathrm{~mm}$ and CAA $<125^{\circ}$ was associated with abnormalities of noctural oximetry. There was no significant association with CSA.

Postoperative studies were carried out on six patients. In three, previously normal noctural oximetry remained unchanged. In two patients frequent but mild dips in desaturation were abolished following transoral removal of the anterior compressive mass and posterior occipitocervical fusion. In one patient preoperative study showed frequent and severe nocturnal desaturations (29 desaturations per hour), decompression resulted in complete resolution of noctural desaturations (fig 2 ).

\section{Discussion}

Respiratory involvement in rheumatoid disease is commonly due to pulmonary fibrosis or pleural disease and there is also an increased incidence of pulmonary infections, bronchiectasis, ${ }^{1}$ micrognathia, temporomandibular and cricoarytenoid joint disease, causing upper airway limitation and obstruction sleep apnoea (OSA). ${ }^{11}$ Disordered breathing during sleep also may be related to lesions of the craniocervical junction such as atlantoaxial dislocation. ${ }^{12} 13$ The patients in the present series were elderly and had severe disabilities related to rheumatoid disease which prevented more detailed study of respiratory function.

In the present series noctural respiratory insufficiency was associated with an increased cervical flexure of the neural axis, vertical migration of the dens and the clivoaxial angle, but not with atlantodental separation. Stevens et $a l^{3}$ showed that sensorimotor long tract signs correlated with combined anterior and posterior compression of the first segment of the cord. Rogers et al ${ }^{14}$ showed that, in patients with vertical translocation of the odontoid process, long tract signs are common and loss of joint position sense is particularly associated with severe compression of the posterior aspect of the spinal cord at the craniocervical junction. In this series only three patients had major compression of the spinal cord. These data suggest that detectable respiratory abnormalities may be associated with deformation of the medulla leading to traumatic neuronal disruption ${ }^{15}$ whilst myelopathy is associated with cord compression. Thus, as in the present study, considerable respiratory impairment may occur without clinical evidence of cord compression.

Our study suggests that in rheumatoid disease, clinically unsuspected disturbance of nocturnal respiration can occur even in the absence of respiratory symptoms, focal brainstem signs or myelopathy. Furthermore in patients studied postoperatively there was resolution of pre-existing nocturnal desaturations following decompression, suggesting a causal relationship between compression at the craniocervical junction and noctural desaturations. In conjunction with routine tests of respiratory function nocturnal oximetry may be a valuable investigation in identifying patients with rheumatoid atlantoaxial disease who are at risk of sudden apnoeic death. Serious nocturnal hypoventilation may also be an important indication for surgical intervention.

1 Jayson $M$ I V, Grennan D $M$. Clinical features of rheumatoid arthritis. In: Weatherall D J, Leadingham J G G, Warrell D A, eds. Oxford Textbook of Medicine, 2nd ed. Oxford: 1987: 16.3-.8

2 Davis F W, Markley H E. Rheumatoid arthritis with death from medullary compression. Ann Int Med 1951; 35: 451-61.

3 Stevens J M, Kendall B E, Crockard H A. The spinal cord in rheumatoid arthritis with clinical myelopathy: a computed myelographic study. $f$ Neurol Neurosurg computed myelographic study

4 Marks J S, Sharp J. Rheumatoid cervical myelopathy. $Q \mathcal{F}$ Med 1981; 50: 307-19.

5 Redlund-Johnell I, Pettersson H. Radiographic measurements of the craniovertebral region designed for evaluation of abnormalities in rheumatoid arthritis using dynamic CT myelography. Neuroradiology 1991 33(Suppl): 413-5.

6 Hunter J V, Stevens J M Kendall B E, Moskovich R, Crockard H A. Radiological measurements of the craniovertebral region designed for evaluation of abnormalities in rheumatoid arthritis. In Redlund-Johnell I, ed. Dislocations of the cervical spine in rheumatoid arthritis. Mislocations of the cervical spine in rheumato

7 Crockard H A, Calder I, Ransford A O. One-stage transoral decompression and posterior fixation in rheumatoid atlanto-axial subluxation. $\mathcal{f}$ Bone $f$ t Surg 1990; 72B: 682-5

8 Fish D R, Howard R S, Wiles C M, Symon L. Respiratory arrest: a complication of cerebellar ectopia in adults. Neurol Neurosurg Psychiatry 1988; 51: 714-5.

9 Warley A R H, Stradling J R, Michell J. Evaluation of the Ohmeda 3700 pulse oximeter. Thorax 1987; 42: 892-6.

10 Redlund-Johnell I. Dislocations of the cervical spine in rheumatoid arthritis. Malmo, 1984 (Thesis)

11 Mahowald M W, Mahowald M L, Bundlie S R, Ytterberg $S$ R. Sleep fragmentation in rheumatoid arthritis. Arth Rheum 1989; 32: 974-81.

12 Hall C W, Danoff D. Sleep attacks: apparent relationship to atlantoaxial dislocation. Arch Neurol 1975; 32: 57-8.

13 Fisher M A, Casey L C, Ellman M H, Perlick S J. Sleep apnea due to odontoid brainstem compression in patient with rheumatoid arthritis. Neurology 1986; 36: 163.

14 Rogers M A, Crockard H A, Moskovich R, et al. Nystagmus and joint position sensation; their importance in posterior occipito-cervical fusion in rheumatoid in posterior occipito-cervical

15 Henderson F C, Geddes J F, Crockard H A Neuropathology of the brainstem and spinal cord in endstage rheumatoid arthritis: implications for treatment. Arch Rheum Dis (In press) 